\title{
AMBERGRIS: PERFUME E SÍNTESE
}

Beatriz Soares Machado Tenius e Evelyn Koeche Schroeder

Departamento de Química Orgânica - Instituto de Química - UFRGS - Av. Bento Gonçalves, 9500 - Campus do Vale - $91501-970$ Porto Alegre - RS

Rossana Angélica Schenato

NPPN - Universidade Federal do Rio de Janeiro - Rio de Janeiro - RJ

Recebido em 13/7/98; aceito em 25/8/99

\begin{abstract}
AMBERGRIS: PERFUME AND SYNTHESIS. Rules for the occurence of the ambergris odor is presented and discussed in terms of the relationship between chemical structure and odor. A general overview of the major approaches in the synthesis of Ambrox ${ }^{\circledR}$, the key ambergris-type compound, is also presented.
\end{abstract}

Keywords: Ambrox ${ }^{\circledR}$; structure-odor relationship; total synthesis.

\section{INTRODUÇÃO}

A teoria mais aceita considera o ambergris uma concreção patológica produzida no intestino da baleia azul (Physeter macrocephalus $L$.). Isto se daria a partir dos alimentos nâo completamente digeridos, o que provocaria uma irritação do seu trato digestivo, levando a formação de abcessos que após rompimento liberariam no mar, na forma de pedras, o material conhecido como ambergris ${ }^{1}$. Este é um dos poucos materiais de origem animal utilizados em perfumaria, possuindo um lugar de destaque dentre os perfumes como o civet (do gato de algália), o almiscar (do veado almiscareiro) e o castóreo (do castor). Seu nome deriva do termo árabe ambar, e do francês, ambre gris (âmbar cinza), que o distigue do âmbar marrom, a resina fossilizada. Este material possui um cheiro sutil que lembra alga marinha, madeira e musgo, mas com doçura particular e um meio tom de inigualável tenacidade ${ }^{2}$.

Desde as primeiras civilizações, até mesmo antes da era Cristã, o homem já estava familiarizado com este material. Inicialmente utilizado em cerimônias religiosas como incenso, o ambergris logo fez parte do cotidiano de reis e nobres por causa de sua fantástica fragrância e misterioso efeito de seu cheiro. $\mathrm{Na}$ Ásia, além de ser usado como droga, era também empregado como condimento em comidas típicas e vinhos ${ }^{3}$.

Devido a suas propriedades restauradoras, o ambergris gozava de grande popularidade na Europa Medieval e fez parte das principais farmacopéias até o fim do último século. A partir do século XX, tornou-se ingrediente dominante na indústria de perfumaria francesa ${ }^{3}$.

Como resultado da ação da luz do sol e flutuação em alto mar, em contato com o oxigênio (geralmente por décadas), o ambergris sofre um processo natural de envelhecimento, durante o qual o cheiro de fezes, parecido com o de peixe morto, desaparece. Ao mesmo tempo que muda em qualidade e se torna mais consistente, sua cor escura desbota ${ }^{3}$.

$\mathrm{O}$ ambergris mais valorizado apresenta coloração do cinza claro ao amarelo creme ou, em casos mais raros, branco calcáreo, possuindo uma complexa e balanceada fragrância que é composta de uma série de notas e subnotas formando um caráter harmonioso.

Seu principal constituínte é o álcool (+)-ambrein (1), que durante o processo de envelhecimento é decomposto pelo oxigênio do ar e pela luz solar levando à formação de seis compostos odoríferos mono-, di- e tri-cíclicos, entre os quais o (-)-ambrox (2), seu constituínte mais importante. Presume-se que os compostos cíclicos 2-4 encontrados na fração volátil se originem da parte bicíclica do ambrein, enquanto os fragmentos menores (5-7) estejam relacionados à parte monocíclica da molécula (Figura 1). ${ }^{4}$<smiles>C=C1CCCC(C)(C)C1CCC(C)=CCCC1C(O)CCC2C(C)CCCC21C</smiles>
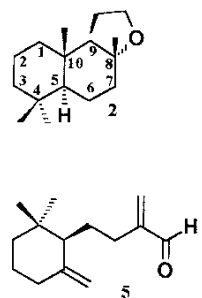
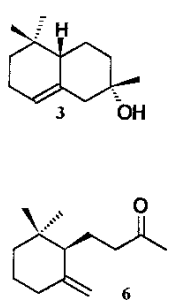
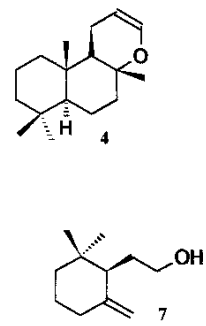

Figura 1: Terpenóides de ocorrência natural isolados do ambergris.

Embora o ambrein (1) seja inodoro, os compostos 2-7 são os principais responsáveis pelas suas raras propriedades olfativas e fixadoras. $\mathrm{O}$ ambergris é usado em perfumaria na forma de sua tintura etanólica que, após um alguns anos de maturação, desenvolve uma fragrância balanceada ${ }^{1}$. A descoberta do ambrein e seus sub-produtos de degradação foi um grande estímulo à pesquisa acadêmica e industrial, especialmente a partir dos anos $50^{5}$.

$\mathrm{O}$ ambergris vem desaparecendo do mercado mundial devido à caça excessiva às baleias no passado e ao contínuo aumento da poluição da costa marítima, tornando ainda mais difícil encontrar materiais de boa qualidade. Até a secreção impura está ficando extremamente rara. Por isso, a indústria de perfumaria está precisando encontrar equivalentes sintéticos para suprir a demanda do produto natural, sendo que o (-)-ambrox (2) é considerado o protótipo sintético mais importante.

Até 100 anos atrás, os perfumes eram totalmente feitos a partir de produtos naturais. As fragrâncias eram obtidas a partir de óleos essenciais extraídos de flores, plantas, raízes e de alguns animais selvagens. Embora os óleos essenciais sejam ainda hoje obtidos a partir dessas fontes naturais, eles têm sido, cada vez mais, substituídos por compostos sintéticos. Hoje em dia, as fragrâncias são utilizadas por um grande número de consumidores.

Talvez o marco principal na história da indústria de perfumaria 
tenha sido o lançamento, em 1921, do famoso perfume Chanel $n^{\circ} 5^{6}$. Esta foi a primeira fragrância a usar compostos orgânicos sintéticos. Seu sucesso imediato levou ao crescente interesse pelo uso de novos materiais em perfumaria. As vantagens destes materiais sintéticos são óbvias: custo, disponibilidade, consistência, estabilidade, etc.

$\mathrm{O}$ ambergris foi usado como base em perfumes nos primeiros tempos da perfumaria, mas teve papel de destaque como nota de fundo ou fixador nas fragrâncias. Quando sua demanda excedeu o suprimento do material natural, houve a necessidade de desenvolvimento de compostos âmbares sintéticos ${ }^{7}$.

\section{ESTRUTURA E ATIVIDADE}

O problema da correlação estrutura/atividade para os compostos químicos é um dos mais importantes na química moderna. A interação dos compostos tipo-ambergris com sua molécula-receptora parece ser um evento extraordinariamente específico. A menor mudança em suas estruturas é, em muitos casos, acompanhada por dramáticas mudanças em suas propriedades olfativas. Assim como no caso da resposta a medicamentos, comunicação entre insetos e percepção dos gostos, o olfato não depende somente da constituição química e configuração relativa de um composto odorífero, mas também de sua configuração absoluta ${ }^{8}$. Interações não covalentes como atração de van der Waals, pontes de hidrogênio, transferência de carga e interações eletrostáticas podem se combinar para produzir uma característica multi dimensional de interação. A extensão de uma ou de outra força pode predominar na interação com um receptor em particular, mas para uma alta afinidade, cada uma delas tem que tender ao máximo ${ }^{9}$. Como o órgão olfativo humano é capaz de distinguir compostos quirais, consideráveis diferenças na qualidade ou potência de odores podem ser percebidas entre compostos enantioméricos.

Por mais de seis décadas, químicos têm pesquisado correlações entre estrutura molecular e odor, com o intuito de sintetizar moléculas com determinadas características odoríferas. Os frutos dessas pesquisas são uma variedade de postulados sobre a relação estrutura-odor (SORs - Structure Odor Relationships). Para cada área de odor existe um tipo de SOR empregada. Em alguns casos, ela está restrita a regras empíricas ou equações, relacionando a intensidade ou semelhança do aroma em alguns exemplos ${ }^{6}$.

A "teoria estereoquímica" mais aceita hoje em dia está baseada na descoberta do cientista inglês John Amoore nos anos $50^{9}$. Amoore especulou que os receptores do nariz, responsáveis em enviar sinais de cada aroma ao cérebro, funcionam como um sitema do tipo "chave e fechadura". A combinação de moléculas odoríferas de diferentes formatos são reconhecidas por sítios específicos (fechaduras), e os sinais enviados ao cérebro, levam à percepção de diferentes cheiros, dependendo da estrutura da molécula. Inicialmente Dyson ${ }^{10}$ e depois Wright $^{11}$ defenderam que este reconhecimento e as semelhanças entre os aromas das moléculas estão relacionados com os movimentos vibracionais das moléculas. Eles acreditam que a gama de receptores do bulbo olfativo são sensíveis às diferentes vibrações das moléculas, e esta informação é recebida pelo cérebro como a "impressão digital" dos aromas.

Quanto à percepção do acento de ambergris, estudos revelaram que este está intimamente relacionado aos elementos estruturais do esqueleto labdano, sendo o ambrox (2) o exemplo típico ${ }^{12}$.

A Regra Triaxial da sensação de odor proposta por Ohloff ${ }^{13}$ em 1971, postula que o odor característico de ambergris ou de qualquer de seus componentes individualmente acontece somente em compostos que possuem um esqueleto biciclo [4.4.0]decano com uma estereoquímica estritamente definida, simbolizada pela Figura 2.

Os odorantes do tipo ambergris mostram uma grande relação estrutural com o esqueleto decalínico com fusão trans de anel (estrutura A da Figura 3).

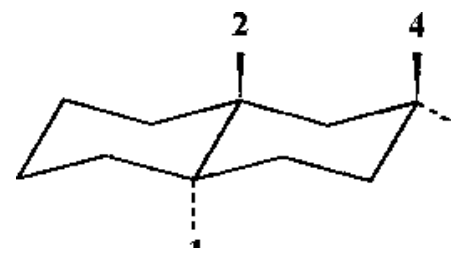

Figura 2. Regra Triaxial da sensação de odor de ambergris.
$\underline{A}$

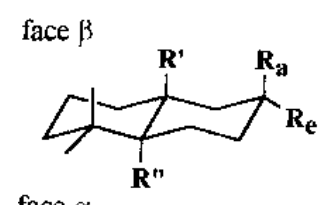

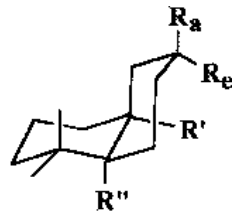

$\underline{B}$
Figura 3. Trans e cis decalinas.

A interação entre as estruturas dos receptores olfativos e o composto odorífero ocorre por uma interação intermolecular de três pontos. Além dos substituintes (ou de hidrogênios) axiais em cabeça de ponte na estrutura $\underline{A}$ ( $R^{\prime}$ e R'), um substituinte axial $R_{a}$ na face $\beta$ serve como um ponto adicional para a ligação não-covalente entre o composto e o receptor. Além disso, a molécula tem que conter pelo menos uma função oxigenada ligada a uma destas posições ${ }^{14}$, como um álcool, um éter ou éster (agindo como o grupo osmofórico) ${ }^{15}$. O derivado decalínico com fusão cis de anel (estrutura $\underline{B}$ ) geralmente não preenche os requerimentos estereoquímicos básicos para ter propriedades do tipo ambergris.

Em 1983 foi descoberto um novo tipo de odorante do tipo ambergris, o cicloexiltetraidrofurano 8 (Figura 4$)^{3}$.

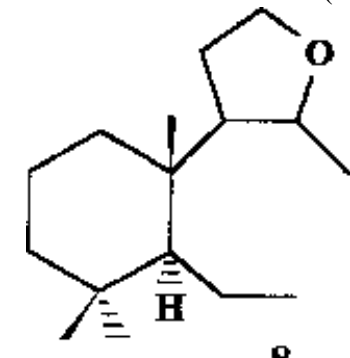

Figura 4: Cicloexiltetraidrofurano de Vlad.

A ausência de um sistema anel decalínico neste composto e a posterior observação de que havia mais compostos que não obedeciam à Regra Triaxial mas possuíam um forte cheiro de ambergris, assim como compostos que se encaixam nesta regra mas não apresentam o cheiro característico, levou a uma pesquisa mais profunda sobre o assunto. Vlad ${ }^{16}$, o autor deste trabalho, concluiu que parâmetros estruturais e estereoquímicos, como ângulo e tamanho da ligação e distância entre certos grupos, não são suficientes para explicar a origem das propriedades do ambergris. Seu trabalho, então, estabeleceu uma correlação estrutura-odor de ambergris em alguns compostos, pela análise de parâmetros eletrônicos e estereoquímicos. Propôs que a origem do odor de ambergris é determinada por certas propriedades eletrônicas de um fragmento estrutural definido. Vlad verificou que todos os odorantes do tipo ambergris possuem um arranjo triangular de certa dimensão (Figura 5) entre três átomos: um sendo oxigênio e outros dois, hidrogênio (axiais, terciários ou alílicos), chamado de Triângulo de ambergris ${ }^{16}$.

De acordo com esta regra, os orbitais atômicos dos dois átomos de hidrogênio $\mathbf{H}_{\mathbf{i}}$ e $\mathbf{H}_{\mathbf{j}}$ têm uma grande contribuição 


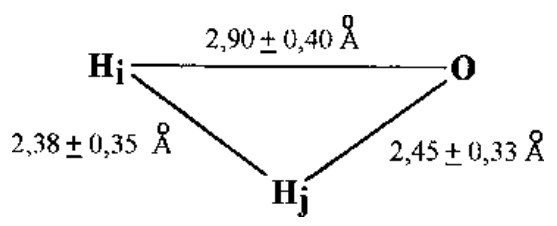

Figura 5. Triângulo de ambergris.

para o orbital molecular ocupado de mais alta energia (HOMO) ou no desocupado de mais baixa energia (LUMO) da molécula toda, que são importantes para a associação com o OM do receptor. Esta abordagem que combina estrutura eletrônica e conformação molecular (topologia) é chamada de método etetrônico-topológico ${ }^{17}$.

Na prática não é necessário fazer cálculos quânticos para determinar o triângulo com distâncias interatômicas definidas formado pelo oxigênio e outros dois hidrogênios. Se uma simples análise estereoquímica for feita e este triângulo estiver presente na molécula, o composto deverá apresentar odor de ambergris.

Existe ainda mais um pré-requisito a ser considerado para o odor de ambergris. Examinando uma série de análogos ativos e inativos de odorantes do tipo ambergris, Winter notou que a acessibilidade estérica do oxigênio do anel tetraidrofurânico do (-)-ambrox é um importante fator estrutural para a atividade odorífera ${ }^{18,19}$. Nos compostos relativamente rígidos, este parâmetro depende da orientação e do ambiente em torno do oxigênio. Winter concluiu que, para um dado composto, a acessibilidade estérica de um grupo funcional depende do ambiente molecular próximo. Para compostos com odor do tipo ambergris, a acessibilidade do átomo de oxigênio, que deve ficar em torno de $8 \mathrm{~A}$, é altamente dependente da conformação e do tamanho da cadeia lateral (Figura 6). Esta observação está de acordo com Ohloff, que também sugeriu, com base na Regra Triaxial, que o átomo de oxigênio em equatorial é o responsável pelo pronunciado cheiro de ambergris do ambrox.
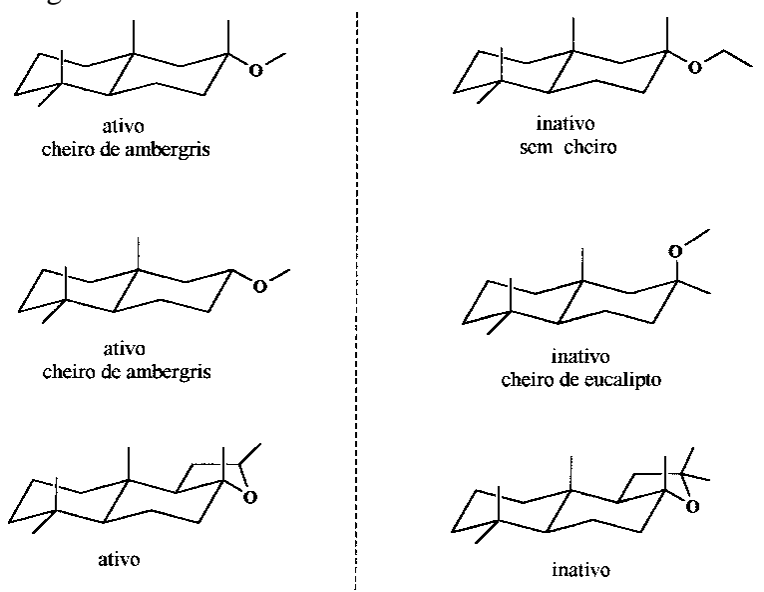

Figura 6: Alguns compostos estudados por Winter.

As três teorias que relacionam estrutura e atividade de compostos com odor de ambergris parecem ser complementares. Mas, apesar de incompleta, a Regra Triaxial do odor de ambergris é a mais ampla, e portanto a mais empregada na modelagem de compostos visando obter novas estruturas com o cheiro do tipo ambergris ${ }^{20,21}$.

A conformação 1,3-synperiplanar/diaxial dos substituintes em C-8 e C-10, assim como a presença da dimetila geminal em C4 e do biciclo [4.4.0] com fusão trans de anel associado como anel tetraidrofurano em posição equatorial, fazem do (-)-ambrox um protótipo (ou um substituto) de todos os compostos com odor de ambergris, tanto estrutural quanto organolepticamente ${ }^{2}$.

\section{SÍNTESES DO AMBROX}

Devido a suas propriedades olfativas e fixativas únicas, o (-)-óxido de norlabdano (2), mais conhecido como Ambrox ${ }^{\circledR}$ (Firmenich), Amberlyn ${ }^{\circledR}$ (Quest) ou Ambroxan ${ }^{\circledR}$ (Henkel), constitui-se hoje no equivalente sintético mais importante em substituição ao ambergris natural. Este composto, que foi sintetizado pela primeira vez por Stoll e Hinder na década dos cinqüenta $^{5}$, mas que somente muito mais tarde foi isolado do próprio ambergris, tem gerado uma grande atividade sintética, tanto em direção ao material racêmico como ao oticamente puro. Grande parte dessas sínteses têm partido de di e sesquiterpenos de ocorrência natural (Figura 7), tais como o (-)sclareol $(\mathbf{9})^{23}$, óxido de manoila $(\mathbf{1 0})^{24},(-)$-ácido labdanólico $(\mathbf{1 1})^{25}$, ácidos comúnicos $(\mathbf{1 2})^{26}$, (-)-ácido abiético $(\mathbf{1 3})^{27},(-)$ drimenol $(\mathbf{1 4})^{28}$, na maioria das vezes tirando partido da estereoquímica correta dos três centros estereogênicos contíguos presentes nestas moléculas.
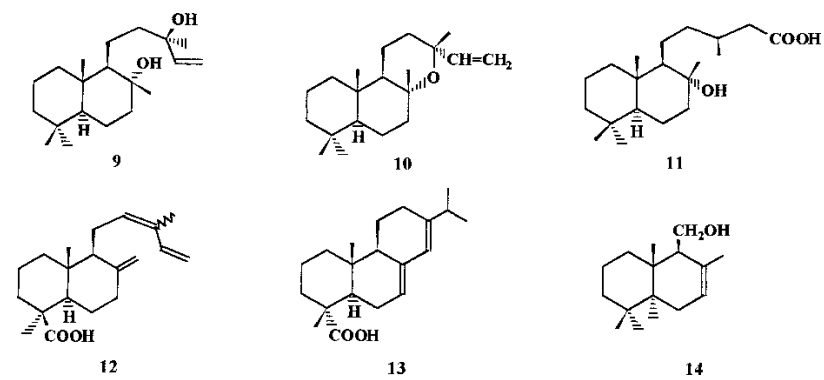

Figura 7. Sesqui e diterpenos naturais usados como material de partida na síntese do ambrox.

Poucas são as sínteses totais relatadas em literatura que constroem os centros estereogênicos presentes na molécula-alvo. Estas partem de monoterpenos como $(S)-(+)$-carvona $(\mathbf{1 5})^{29}$ e thujona $(\mathbf{1 6})^{30,31}$, e também de polienos como, por exemplo, a geranilacetona $(\mathbf{1 7})^{32}$, ácido farnésico $(\mathbf{1 8})$ e derivados ${ }^{33}$.

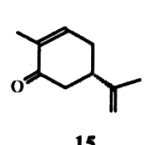

15

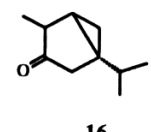

16

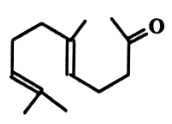

17

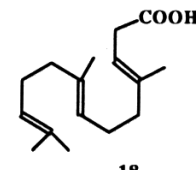

18
Figura 8. Monoterpenos e polienos usados como material de partida na síntese do ambrox.

A análise das várias rotas sintéticas elaboradas para a preparação de $\mathbf{2}$ mostram a existência de intermediários-chave comuns, como o diol 19 ou o álcool 20, ocorrendo na imensa maioria dos casos.<smiles>C[C@H]1CCC[C@@]2(C)C(CCO)[C@](C)(O)CC[C@@H]12</smiles>

19

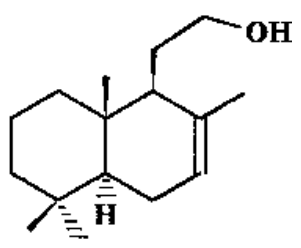

20
Figura 9. Intermediários mais comuns nas sínteses do Ambrox.

A elaboração final, para a construção do anel tetraidrofurânico a partir destes intermediários é a ciclização ácido catalisada do carbocátion terciário 21, comum a ambos. Quando se utiliza o sistema APTS/MeNO${ }_{2}$ e a reação é feita 
a $70-90^{\circ} \mathrm{C}$, é obtida uma mistura de $\mathbf{2}$ e $\boldsymbol{e p i - 2}$ na proporção $1,5: 1$, enquanto que à temperatura ambiente há formação quase exclusiva de 2 (proporção 20:1 estimada por RMN de $\left.{ }^{1} \mathrm{H}\right) .{ }^{34} \mathrm{~A}$ preferência pelos produtos obtidos nas duas temperaturas concorda com o estabelecido, que o anel tetraidrofurano com fusão trans (2) é o isômero cinético (favorecido à t.a.) e o anel com fusão cis (epi-2) é o termodinâmico (favorecido a temperaturas mais altas) ${ }^{31,53}$.
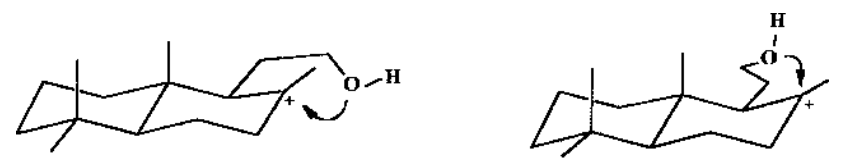

21

Figura 10. Ciclização ao (-)-ambrox.

\section{SÍNTESES A PARTIR DE DITERPENOS}

O (-)-sclareol $(9)^{35}$, um produto natural obtido como metabólito secundário da Salvia sclarea (uma planta de origem russa) e também a partir de novas linhas da planta do tabaco (a Nicotina glutinosa ${ }^{36}$ ), é o representante deste tipo de material de partida mais utilizado para a síntese do (-)-ambrox, inclusive em escala industrial. Como no caso de outros diterpenos utilizados nas sínteses de (-)-2, o intermediário 19 é obtido pela degradação oxidativa das cadeias laterais destes compostos com o uso de agentes oxidantes como o ozônio ${ }^{37}$, peróxidos ${ }^{38}$, trióxido de cromo $^{39}$, permanganato de potássio ${ }^{40}$ e até microorganismos ${ }^{41}$. Mais recentemente foi utilizado, também, óxido de rutênio catalítico ${ }^{42}$.

Um exemplo de síntese bem sucedida utilizando esta metodologia foi realizada por Paul Martre ${ }^{42}$, que obteve o (-)ambrox em três etapas a partir do (-)-sclareol com rendimento total de $79 \%$ (Esquema 1). Inicialmente a cadeia lateral do sclareol é clivada, com perda de quatro átomos de carbono, para dar uma mistura de 82:18 do (-)-acetoxiácido 22 e do (+)sclareolídeo 23 com $88 \%$ de rendimento (variante a). Se, entretanto, a reação é feita em condições básicas, na presença de catalisador de transferência de fase, o (+)-sclareolídeo 23 é obtido como único produto com $54 \%$ de rendimento, sendo que o intermediário (-)-22 é saponificado e cicliza a 23 (variante b). Tanto 23 como 22 podem ser reduzidos com $\mathrm{LiAlH}_{4}$ ao diol 19, que é ciclizado ao (-)-ambrox nas condições usuais.

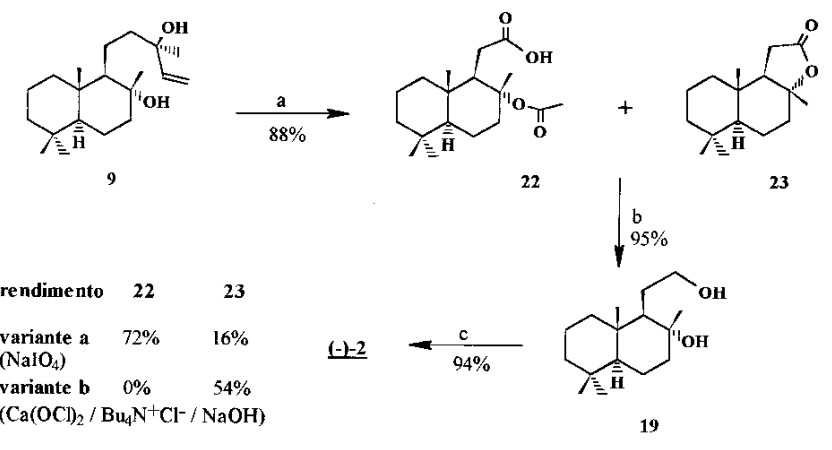

a) $\mathrm{RuCl}_{3} 3 \mathrm{H}_{2} \mathrm{O}, \mathrm{CCl}_{4}, \mathrm{CH}_{3} \mathrm{CN}, \mathrm{H}_{2} \mathrm{O}, 40^{\circ} \mathrm{C}$; b) $\mathrm{LiAlH}_{4}, \mathrm{Et}_{2} \mathrm{O}, 25^{\circ} \mathrm{C}$; cl NaH, $\mathrm{TsCl}, \mathrm{CH}_{2} \mathrm{Cl}_{2}, 25^{\circ} \mathrm{C}$

Esquema 1. Síntese do (-)-ambrox de Paul Martre.

\section{SÍNTESE A PARTIR DE SESQUITERPENOS}

O único representante deste tipo de material de partida para a síntese do (-)-ambrox é o drimano (-)-drimenol (14) ${ }^{28}$ (Esquema 2), principal componente das cascas da árvore da espécie Drimys witeri. Neste caso, o intermediário 19 foi obtido através da modificação da cadeia lateral deste sesquiterpeno, conforme mostrado no esquema 2: a homologação da cadeia lateral de 14 é conseguida via substituição do derivado mesilado por $\mathrm{NaCN}$ em condições de transferência de fase. A posterior hidrólise da nitrila seguindo o método de Huang-Minlon, leva ao ácido 26 que lactoniza ao epi-12nor-ambrenolídeo 27, precursor direto da molécula-alvo. A redução à 19 e posterior ciclização em condições usuais leva ao (-)-ambrox com $25 \%$ de rendimento total.
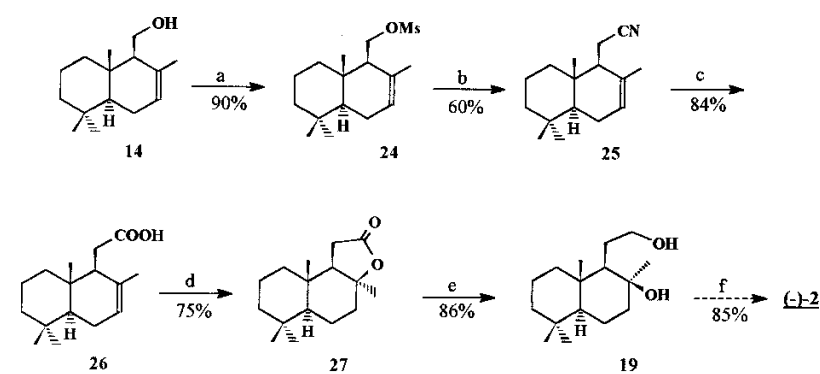

a) MsCl; Py, t.a., 40 h; b) NaCN, Adogen 464, tolueno, $1000 \mathrm{C}$; c) KOH, $\mathrm{HOCH}_{2} \mathrm{CH}_{2} \mathrm{OH}$, refluxo, 24h; d) APTS, $\mathrm{CH}_{2} \mathrm{Cl}_{2}$, refluxo, hh; e) $\mathrm{LiAlH}_{4}$, THF, t.a., 3h; f) A.Prs, $\mathrm{MeNO}_{2}$, t.a., 3h.

Esquema 2. Síntese do (-)-ambrox de Cortés.

\section{SÍNTESES A PARTIR DE MONOTERPENOS}

Quando diterpenos são utilizados para a preparação do (-)ambrox, os quatro centros estereogênicos da molécula-alvo, com a configuração correta, já estão presentes no material de partida. Ao contrário, quando se utiliza monoterpenos, a abordagem sintética deve prever a sua construção, de forma estereosseletiva, o que torna a tarefa sintética muito mais complexa. A utilização de monoterpenos oticamente ativos como a carvona (15) ou a thujona (16) oferecem a oportunidade do aproveitamento de seus centros estereogênicos para induzir a estereosseletividade das etapas subseqüentes, o que pode ser de grande utilidade sintética.

Esta estratégia foi seguida por de $\operatorname{Groot}^{29}$ para a preparação de (-)-2. Sua transformação no álcool bicíclico 20, intermediário-chave também nesta síntese, se deu a partir da octalona 29, preparada de forma estereosseletiva a partir da S-(+)carvona, conforme mostrado no Esquema 3. O rendimento final de (-)-2 nesta síntese de 11 etapas foi de $10 \%$.

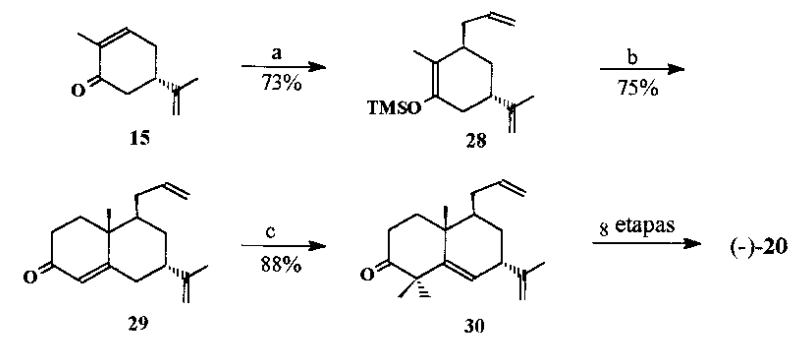

a) (alii)MgCl, Mes $\mathrm{S} . \mathrm{CuBr}$, TMSCl, $\mathrm{HMPA}, \mathrm{THF},-100 \circ \mathrm{C}$ (d.e.=88\%); b) 1- $\mathrm{MVK}, \mathrm{BF}_{3} . \mathrm{Et}_{2} \mathrm{O}$, i-PrOH, $\mathrm{CH}_{2} \mathrm{Cl}_{3}$, $\mathrm{CH} 3 \mathrm{NO}_{2}, 65^{\circ} \mathrm{C}$ (d.e. $=94 \%$ ); 2- NaOMe, MeOH; c) MeI, ${ }^{\mathrm{B}} \mathrm{HOK}, \mathrm{BuOH}$

Esquema 3. Síntese de de Groot para o intermediário 20.

A thujona (16), obtida a partir do óleo essencial extraído do resíduo de galhos e folhas do cedro vermelho (Thuja plicata Donn), através do seu sistema rígido biciclo (3.1.0) hexano, leva a produtos regio e estereosseletivos quando submetida a reações como anelação de Robinson, gerando bons synthons quirais para a síntese de terpenos e esteróides, como por exemplos as 
octalonas 35 e 36, utilizadas por Kutney para a síntese do (-)ambrox. Em sua primeira abordagem sintética ${ }^{30}$ (Esquema 4), a octalona 31 é transformada no diol 34, o qual sofre ciclização em meio ácido para gerar o produto alvo, num processo em sete etapas e com um redimento total de $9.5 \%$.

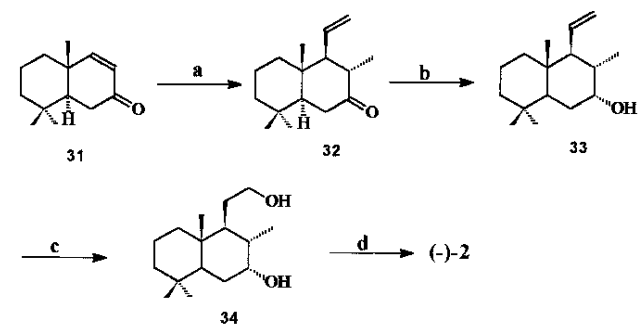

a) 1- $\mathrm{CH}_{2}=\mathrm{CHMgBr}$, Cul, THF; 2- LDA, DME, $\mathrm{CH}_{3}$; b) 1-LDA,THF; 2- $\mathrm{PhSeCl}$; $3-\mathrm{H}_{2} \mathrm{O}_{2}, \mathrm{pir}, \mathrm{CH}_{2} \mathrm{Cl}_{2} ; 4-\mathrm{Li}_{1}, \mathrm{NH}_{3}$, $\mathrm{NH}_{4} \mathrm{Cl}$; 5- L-Selectriäe, THF; c) 1- $\mathrm{BH}_{3}-\mathrm{THF}^{2} ; 2-\mathrm{H}_{2} \mathrm{O}_{2} ;$ d) TsOH, Tol., $80^{\circ} \mathrm{C}$

Esquema 4. Síntese de Kutney para o (-)-Ambrox a partir da octalona 31 .

A segunda síntese,${ }^{31}$ de 1997 (Esquema 5), mostra a utilização da octalona 35 como material de partida. Inicialmente a transposição da carbonila da enona leva a formação de $\mathbf{3 6}$. A alquilação deste composto e a sua cetalização em condições próticas leva a formação preferencial do produto com a estereoquímica correta, quando se aplicam condições de equilibração termodinâmica. Os passos subsequentes desta síntese levam a preparação do intermediário 19 , novamente utilizado na preparação do (-)-ambrox.

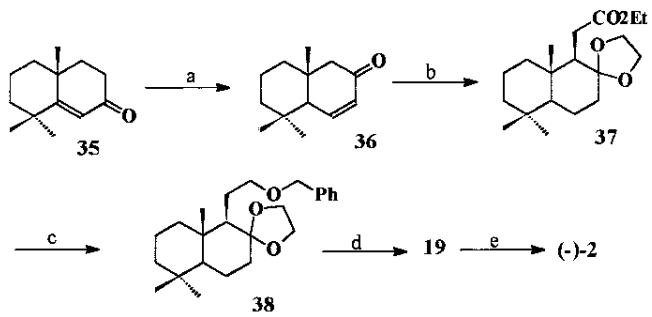

1- $\mathrm{Mn}\left(\mathrm{OAC}_{3} . \mathrm{H}_{2} \mathrm{O}\right.$, benzeno; 2- LiAlH4, THF; 3- 1,2 equiv. TsOH, THF; b) 1- $\mathrm{LDA}, \mathrm{ICH}_{2} \mathrm{CO}_{2} \mathrm{Et} ; 2-\mathrm{H}_{2} / \mathrm{Pd}-\mathrm{C}$; $3-$ 1,2-bis-(TMSO)etano, TMS-triflato; c) 1- $\mathrm{LiAlH}_{4}$; 2- $\mathrm{PhCH}_{2} \mathrm{Br}, \mathrm{n}$-BuiNl; d) 1- $\mathrm{HCl}$; 2- $\mathrm{MeLi}$, $\mathrm{CeCl}_{3} ; 3-\mathrm{H}_{2} / \mathrm{Pd}$ $\mathrm{C} ;$ e) $\mathrm{p}-\mathrm{T} \mathrm{SOH}, \mathrm{CH}_{3} \mathrm{NO}_{2}$

Esquema 5. Síntese de Kutney para o (-)-ambrox a partir da octalona 35.

\section{SÍNTESE A PARTIR DE POLIENOS}

A ciclização biomimética de polienos tem sido muito aplicada na síntese de terpenóides policíclicos e esteróides por causa da sua alta esterosseletividade ${ }^{43}$. Quanto à preparação do ambrox racêmico, várias sínteses formais e totais baseadas na ciclização de polienos acíclicos e cíclicos como o ácido farnésico, monofarnésico e seus derivados ${ }^{44,45,46}$ têm sido propostas. Entre os agentes ciclizantes empregados nesta metodologia estão o $\mathrm{SnCl}_{4}{ }^{43,47}, \mathrm{CF}_{3} \mathrm{COOH}^{48}, \mathrm{HCOOH}$ $\mathrm{H}_{2} \mathrm{SO}_{4}{ }^{49} \mathrm{e}$, mais recentemente, os superácidos $\mathrm{FSO}_{3} \mathrm{H}^{45,50}$ e $\mathrm{ClSO}_{3} \mathrm{H}^{33}$. Nestas ciclizações, a estereoquímica das duplas ligações é fundamental para a garantia da formação de um anel trans-decalínico.

Dos produtos de partida mais utilizados nestas ciclizações catalisadas por ácido estão a geranilacetona $(\mathbf{1 7})^{32}$ e a dihidro- $\beta$ ionona $(\mathbf{3 9})^{51,52,53}$, obtida pela hidrogenação da $\beta$-ionona com hidreto de terc-butil estanho e AIBN catalítico. Em 1992, Snowden ${ }^{45}$ propôs a síntese do $( \pm)$-ambrox e seus diastereoisômeros, pela ciclização biomomética de 17 e 39 (Esquema 6). A homologação por adição de três carbonos às cadeias laterais destes compostos é feita via reação com ilídeos de fósforo. Os hidróxipolienos 40 e 41 são ciclizados com catálise de superácidos para resultar no $( \pm)$-ambrox com baixos rendimentos.
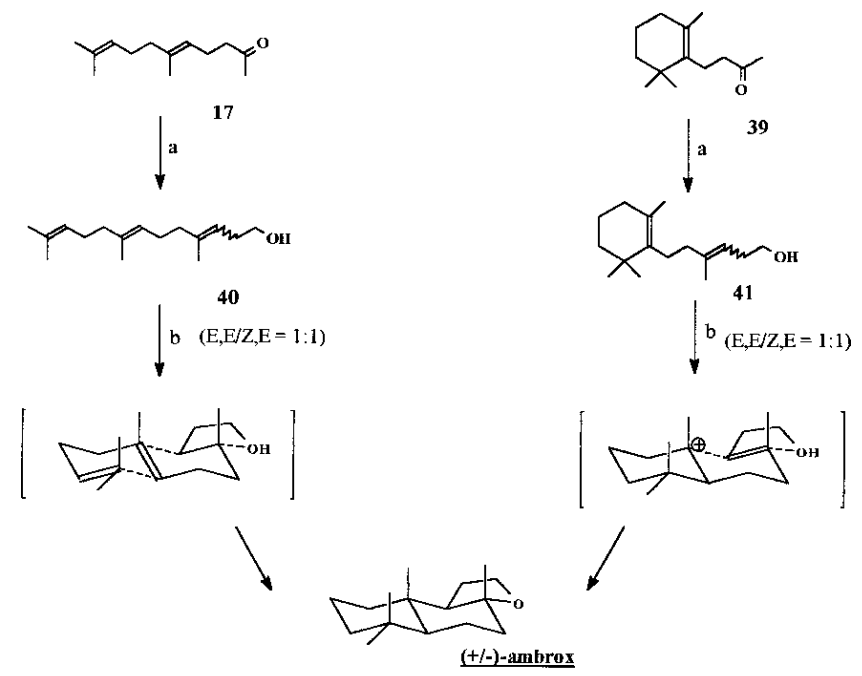

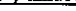

a) $\left[\phi_{3} P\left(\mathrm{CH}_{2}\right]_{3} \mathrm{OH}\right]^{+}+\mathrm{Br}, 2$ eq. ${ }^{\mathrm{B}} \mathrm{BuLi}, \mathrm{THF}$; b) $\mathrm{HSO}_{3} \mathrm{~F}, \mathrm{PTNO}_{2},-90{ }^{\circ} \mathrm{C}$.

Esquema 6. Ciclização biomimética dos hidroxidopolienos 40 e 41.

Outra alternativa para a obtenção de intermediários sintéticos enantiomericamente puros para a preparação do (-)ambrox foi o uso de catálise enzimática para a resolução do intermediário cíclico obtido a partir do acetato homofarnésico $\mathbf{4 2}^{33}$ (Esquema 7). O substrato racêmico $\mathbf{4 3}$ foi submetido à resolução enzimática com lipase PS-30 (Pseudomonas $s p$ ) resultando no acetato (-)-44 com $97 \%$ de excesso enantiomérico. $\mathrm{O}$ aumento da cadeia lateral foi efetuado na forma indicada no Esquema 7, levando a obtenção do (-)-ambrox via intermediário 19, numa síntese em 7 passos a partir de 43 com $35 \%$ de rendimento.
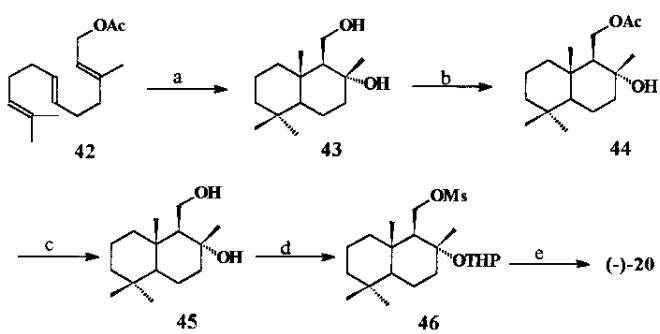

a) $\mathrm{ClSO}_{3} \mathrm{H}$; bj lipase PS-3O, acetato de vinila, 1d; c) $\mathrm{LiAlH}_{4}, \mathrm{Et} 2 \mathrm{O}$; dl 1- $\mathrm{MsCl}$, Pir.; 2- $\mathrm{DHP}$, $\mathrm{TsOH}_{\mathrm{SO}} \mathrm{CH}_{2} \mathrm{Cl}_{2}$; e) 1NaCN, 18-Crown-6, DMSO; 2- Dibal, $\mathrm{CH}_{2} \mathrm{Cl}_{2} ; 3$ - $\mathrm{LiAlH}_{4}, \mathrm{Et}_{2} \mathrm{O}$

Esquema 7. Síntese do (-)-ambrox a partir do acetato homofarnésico (42), com um passo de resolução enzimática.

Recentemente, uma nova síntese do (-)-Ambrox foi publicada ${ }^{54}$ beseada na funcionalização enzimática de um sínton quiral do tipo decalina.

\section{CONCLUSÕES}

Devido as suas propriedades fixativas únicas e ao seu odor especial, o (-)-ambrox, constitui-se hoje no mais importante equivalente sinético do ambergris natural, que por razões de pesca predatória e da alta demanda mundial por este produto de química fina, acha-se em extinção. A principal fonte de matéria prima para a preparação industrial do ambrox, a Salvia sclarea, também se acha em vias de desaparecimento, o que 
tem levado a um grande esforço sintético no intuito do planejamento de sínteses economicamente eficientes e elegantes.

\section{REFERÊNCIAS}

1. Anonis, D. P.; Perfumer \& Flavorist 1995, $20,7$.

2. Ohloff, G.; "The Fragrance of Ambergris" in "Fragrance Chemistry - The Science of the Sense of Smell”; Theimer E. T., Ed.; Academic Press: New York, 1982, 535.

3. Sell, C.; Chem. \& Ind. 1990, 516.

4. Ohloff, G.; Schulte-Elte, K. H.; Müller, B. L.; Helv. Chim. Acta 1977, 60, 2763.

5. Stoll, M.; Hinder, M.; Helv. Chim. Acta 1950, 33, 1251.

6. Rossiter, K. J.; Chem. Rev. 1996, 96, 3201.

7. Anonis, D. P.; Perfumer \& Flavorist 1996, 21, 03.

8. Gautier, A.; Vial, C.; Morel, C.; Lander, M.; Näf, F.; Helv. Chim. Acta 1987, 70, 2039.

9. Amoore, J. E.; Perfum. Essent. Oil Rec. 1952, 43, 321. 10. Dyson, G. M., Perfum. Essen. Oil Rec. 1937, $28,13$.

11. Wright,R.H., J. Appl. Chem. 1954, 4, 611.

12. Ohloff, G.; Giersch, W.; Pickenhagen, W.; Furrer, A.; Frei, B.; Helv. Chim. Acta 1985, 68, 2022.

13. Ohloff, G.; in "Gustation and Olfaction"; Ohloff, G. and Thomas, A. F., Eds.; Academic Press: London, 1971, pp 178.

14. Ohloff, G.; Vial, C.; Wolf, H. R.; Job, K.; Jégou, E.; Polonsky, J.; Lederer, E.; Helv. Chim. Acta 1980, 63, 1932.

15. Näf, F.; Vial, C.; Thommen, W.; Helv. Chim. Acta 1989, 72, 1989.

16. Vlad, P. F.; Bersuker, I. B.; Dimoglo, A. S.; Gorobachov, M. Y.; Koltsa, M. N.; Nouv. J. Chim. 1985, 9, 211.

17. Vlad, P. F.; Dimoglo, A. S.; Shvets, N. M.; Coltsa, M. N.; Güzel, Y.; Saraçoglu, M.; Saripinar, E.; Patat, S.; New J. Chem. 1995, 19, 1217.

18. Winter, B.; Helv. Chim. Acta 1989, 72, 1278.

19. Winter, B.; Pure \& Appl. Chem. 1990, 62, 1377.

20. Ohloff, G.; Näf, F.; Decorzant, R.; Thommen, W.; Sundt, E.; Helv. Chim. Acta 1973, 56, 1414.

21. Ohloff, G.; Giersch, W.; Schulte-Elte, K.; Vial, C.; Helv. Chim. Acta 1976, 59, 1140.

22. Ohloff, G.; Escher, S; Giersch, W.; Niclass, Y.; Bernardinelli, G.; Helv. Chim. Acta 1990, 73, 1935.

23. Coste-Manière, I. C.; Zahra, J. P.; Waegell, B.; Tetrahedron Lett. 1988, 29, 1017.

24. Cambie, R. C.; Joblin, K. N.; Preston, A. F; Aust. J. Chem. 1971, 24, 583.

25. Urones, J. G.; Pedrero, A. M.; Teresa, J. P.; Barcala, P.B.; Tetrahedron Lett. 1985, 26, 5717.

26. Barrero, A. F.; Altarejos, J.; Manzaneda, E. J. A.; Ramos, J. M.; Salido, S.; Tetrahedron 1993, 49, 9525.
27. Ohno, M.; Koyama, H.; Kaku, Y.; Tetrahedron Lett. 1987, $28,2863$.

28. Cortés, M. J.; Sierra, M. G.; Rúveda, E. A.; López, J.T.; Heterocycles 1987, 26, 2801.

29. de Groot, A.; Haaksma, A. V.; Swarts, H. J.; Jansen, B. J. M.; Tetrahedron 1994, 50, 10073.

30. Kutney, J. P.; Chen, Y. H.; Can. J. Chem. 1994, 72, 1570.

31. Kutney, J. P.; Cirera, C., Can. J. Chem. 1997, 75, 1136

32. Mori, K.; Tamura, H.; Liebigs Ann. Chem. 1990, 361.

33. Oritani, T.; Tanimoto, H.; Tetrahedron: Asymm. 1996, 7, 1695.

34. Barrero, A. F.; Altarejos, J.; Manzaneda, E. J. A.; Ramos, J. M.; Salido, S.; Tetrahedron 1993, 49, 6251.

35. Ehret, C.; $9^{\text {th }}$ Int. Congr. Essent. Oils, Singapore, 1983 , 3, 77.

36. Patent EP 363,774 (priority 12.10.88).

37. Barton, D. H. R.; Taylor, D. K.; Tse, C. I; Tetrahedron Lett. 1994, 35, 9505.

38. Christenson, P.A.; Tetrahedron 1988, 44, 1925.

39. Näf, F.; Decorzant, R.; Vial, C.; Tetrahedron 1987, 43, 1871.

40. Ruzicka, L.; Seidel, C. F.; Engel, L. L.; Helv. Chim. Acta 1942, 25, 641.

41. U. S. Patent 4, 798, 799 (17.01.89).

42. Martres, P.; Perfetti, P.; Zahra, J. P.; Waegell, B.; Giraudi, E.; Petrzilka, M.; Tetrahedron Lett. 1993, 34, 629.

43. Saito, A.; Matsushita, H.; Kaneko, H.; Chem. Lett. 1983, 729.

44. Snowden, R. L.; Linder, S. M.; Tetrahedron Lett. 1991, 32,4119 .

45. Snowden, R. L.; Eichenberger, J. C; Linder, S. M.; Sonnay, P.; Vial, C.; Schulte-Elte, K. H; J. Org. Chem. 1992, 57, 955.

46. Barco, A.; Benetti, S.; Bianchi, A.; Casolari, A.; Guarneri, M.; Pollini, G. P.; Tetrahedron 1995, 51, 8333.

47. Saito, A.; Matsushita, H.; Tsijino, Y.; Kaneko, H.; Chem. Lett. 1981, 757.

48. Matsui, M.; Kawanobe, T.; Kogami, K.; Agric. Biol. Chem. 1986, 50, 1475.

49. Lucius, G.; Angew. Chem. 1956, 68, 247.

50. Vlad, P. F.; Pure Appl. Chem. 1993, 65, 1329.

51. Barrero, A. F.; Altarejos, J.; Manzaneda, E. J. A.; Ramos, J. M.; Salido, S.; J. Org. Chem. 1996, 61, 2215.

52. Snowden, R. L.; Eichenberger, J. C; Giersh, W.; Thommen, W.; Schulte-Elte, K. H; Helv. Chim. Acta 1993, 76, 1608

53. Büchi, G.; Wüest, H.; Helv. Chim. Acta 1989, 72, 996.

54. Akita, H.; Nozawa, M.; Shimizu,H., Tetrahedron: Asymmetry 9, 1789. 